CRYSTALLOGRAPHIC COMMUNICATIONS

ISSN 2056-9890

Received 25 February 2019

Accepted 26 March 2019

Edited by W. T. A. Harrison, University of Aberdeen, Scotland

Keywords: crystal structure; memantine-carboxyborane; CORCB; memantine; adamantane.

CCDC reference: 1905840

Supporting information: this article has supporting information at journals.iucr.org/e

\section{Crystal structure of memantine-carboxyborane}

\author{
Theppawut I. Ayudhya, ${ }^{a}$ Arnold L. Rheingold ${ }^{b}$ and Nin N. Dingra ${ }^{a} *$
}

a Department of Chemistry, University of Alaska Anchorage, Anchorage, AK 99508, USA, and ${ }^{\mathbf{b}}$ Department of Chemistry,
University of California-San Diego, La Jolla, CA 92093, USA. *Correspondence e-mail: ndingra@alaska.edu

The synthesis and crystal structure of the title compound, $\mathrm{C}_{13} \mathrm{H}_{24} \mathrm{BNO}_{2}$ [systematic name: 3,5-dimethyladamantanylamine-boranecarboxylic acid or $\mathrm{N}$-(carboxyboranylidene)-3,5-dimethyladamantan-1-amine], derived from the anti-Alzheimer's disease drug memantine is reported. The $\mathrm{C}-\mathrm{N}-\mathrm{B}-\mathrm{CO}_{2}$ unit is almost planar (r.m.s. deviation $=0.095 \AA$ ). The extended structure shows typical carboxylic acid inversion dimers linked by pairwise $\mathrm{O}-\mathrm{H} \cdots \mathrm{O}$ hydrogen bonds $[\mathrm{O} \cdots \mathrm{O}=2.662(3) \AA]$. The amino group forms a weak $\mathrm{N}-\mathrm{H} \cdots \mathrm{O}$ hydrogen bond $[\mathrm{N} \cdots \mathrm{O}=3.011$ (3) $\AA$ ], linking the dimers into [001] chains in the crystal. Highly disordered solvent molecules were treated using the SQUEEZE routine of PLATON [Spek (2015). Acta Cryst. C71, 9-18], which treats the electron density as a diffuse contribution without assignment of specific atom locations. A scattering contribution of 255 electrons was removed. The crystal studied was refined as a two-component twin.

\section{Chemical context}

Memantine is a drug used for the treatment of mild and moderate-to-severe Alzheimer's disease as an inhibitor for $N$-methyl-D-aspartate (NMDA) receptors. As a result of its property as a low-affinity, open-channel blocker, memantine does not substantially interfere with normal synaptic activity, thereby reducing side effects. This has led to clinical trials for other neurological disorders (Bullock, 2006; Lipton, 2005; Olivares et al., 2012; Parsons et al., 2007). While memantine in its hydrochloride form is useful in various treatment methods, some modifications were done on this drug to optimize the desired concentration in the system. As a means to preventing drug degradation, memantine has been further processed in a mixture with other compounds (McInnes et al., 2010; Plosker, 2015). The one-week extended release formula by Lyndra Therapeutics is currently under clinical trial phase I (clinicaltrials.gov, NCT03711825). Though efforts to maintain the long-term stability of memantine are underway, chemical modification of the memantine structure itself is rarely reported. Our attempt was to mask the compound with an additional moiety that can be removed under certain conditions, therefore releasing the drug. With this goal, memantinecarboxyborane was synthesized since the carboxyborate group is known to decompose into carbon monoxide and boric acid, leaving the drug molecule itself (Ayudhya et al., 2017, 2018). The single crystal structure of the said compound, (I), was solved and its features are described in this report.

\section{Structural commentary}

The molecular structure of (I) is shown in Fig. 1. The C2-N1$\mathrm{B} 1-\mathrm{C} 1 / \mathrm{O} 1 / \mathrm{O} 2$ fragment is almost planar (r.m.s. deviation = 
$0.095 \AA$ ) and the $\mathrm{C}$ atoms bonded to the $\mathrm{B}$ and $\mathrm{N}$ atoms take on an anti orientation $\left[\mathrm{C} 1-\mathrm{B} 1-\mathrm{N} 1-\mathrm{C} 2=173.5(3)^{\circ}\right]$. The stereogenic centres in the adamantane unit were assigned as C4 $S$ and C8 $R$ in the arbitrarily chosen asymmetric unit but crystal symmetry generates a racemic mixture. The bond lengths $[\mathrm{C} 1-\mathrm{O} 1=1.340(4), \mathrm{C} 1-\mathrm{O} 2=1.227$ (4) $\AA$ ] of the carboxylic acid group are in agreement with the data for related carboxylic acids and known amine-carboxyboranes (Gavezzotti, 2008; Spielvogel et al., 1980; Vyakaranam et al., 2002; Ayudhya et al., 2017). The $\mathrm{C}-\mathrm{C}-\mathrm{C}$ bond angles of the adamantine cage fall within the expected ranges and the $\mathrm{N} 1-$ $\mathrm{C} 2$ bond length at 1.504 (4) $\AA$ is comparable with previously reported values in aminoadamantane structures (Donohue \& Goodman, 1967; Chacko \& Zand, 1973).

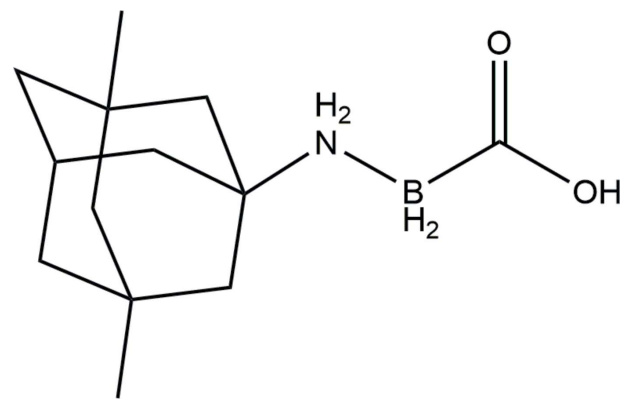

\section{Supramolecular features}

A dimer is observed between the two memantine-carboxyborane molecules formed through conventional hydrogen bonding between the carboxylic acid moieties (Fig. 2). The hydrogen-bond length listed in Table $1[\mathrm{O} 1 \cdots \mathrm{O} 2=$ $2.662(3) \AA]$ is consistent with the hydrogen-bond geometries found in carboxyborane dimers such as ammonia-carboxyborane [2.668 (2) А̊; Spielvogel et al., 1980], mophorlinecarboxyborane [2.712 (4) А; Vyakaranam et al., 2002] and trimethylamine-carboxyborane [2.714 ̊̊; Spielvogel et al., 1976]. In (I), these dimers form an extended structure through $\mathrm{N} 1-\mathrm{H} 1 B \cdots \mathrm{O} 1$ links $(\mathrm{O} 1$ is the protonated oxygen atom of the carboxylic acid), to form [001] chains. This motif has also

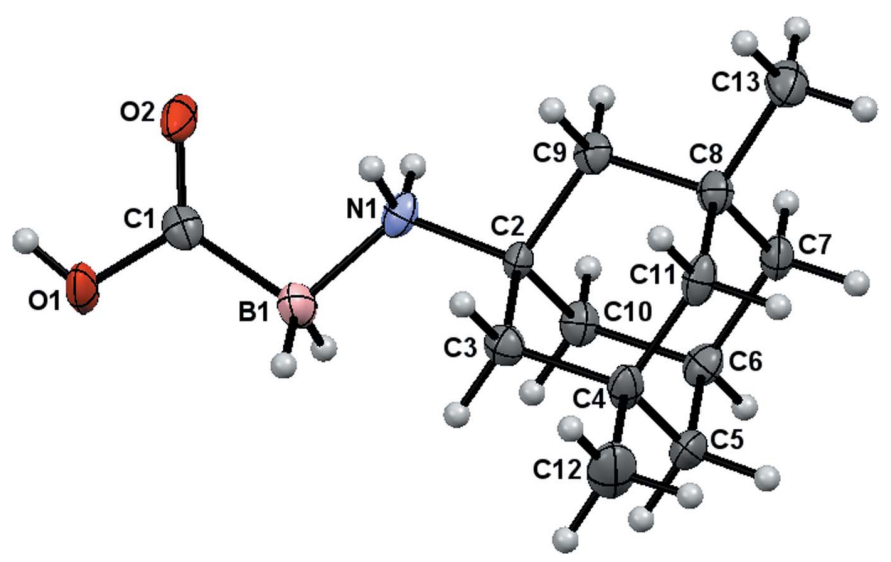

Figure 1

The molecular structure of (I) with displacement ellipsoids drawn at the $50 \%$ probability level.
Table 1

Hydrogen-bond geometry $\left(\AA{ }^{\circ}\right)$.

\begin{tabular}{lllll}
\hline$D-\mathrm{H} \cdots A$ & $D-\mathrm{H}$ & $\mathrm{H} \cdots A$ & $D \cdots A$ & $D-\mathrm{H} \cdots A$ \\
\hline $\mathrm{O} 1-\mathrm{H} 1 \cdots \mathrm{O} 2^{\mathrm{i}}$ & 0.84 & 1.82 & $2.662(3)$ & 176 \\
$\mathrm{~N} 1-\mathrm{H} 1 B \cdots \mathrm{O} 1^{\mathrm{ii}}$ & 0.91 & 2.11 & $3.011(3)$ & 171 \\
\hline
\end{tabular}

Symmetry codes: (i) $-x+1,-y+2,-z+2$; (ii) $x,-y+2, z-\frac{1}{2}$.

been reported previously in ammonia-carboxyborane, trimethylamine-carboxyborane, dimethylamine-carboxyborane and methylamine-carboxyborane (Spielvogel et al., 1980). The adjacent dimers shown in Fig. 2 indicates that the planes of the carboxylic acids are not parallel, but twisted by $76.5^{\circ}$ from each other.

Assessment of available crystal structures deposited with the Cambridge Structural Database (Version 5.39; Groom et al., 2016) indicates that not all amine-carboxyboranes form dimers during crystallization. While some amine-carboxyboranes described above are dimers, others such as piperidine-carboxyborane and hexamethylenetetraminecarboxyborane do not form dimers, suggesting that the aminegroup interaction may influence the overall packing (Rana et al., 2002; Ayudhya et al., 2017). The extended structure of (I) is shown in projection down the $b$ - and $c$-axis directions in Fig. $3 a$ and $3 b$, respectively. No other contacts beyond the hydrogen bonds already mentioned are observed in this packing. Although the dimers appear to be parallel in Fig. $3 a$, the twisted planes of hydrogen bonds are better represented in Fig. $3 b$.

\section{Database survey}

The memantine structure in its free (unprotonated) base form is not found in the literature, although the hydrochloride salt with water molecules of crystallization has been solved (Lou et al., 2009). Another memantine crystal structure reported was in a clathrate form with cucurbit[7]uril where memantine is completely bound within the cavity (McInnes et al., 2010).

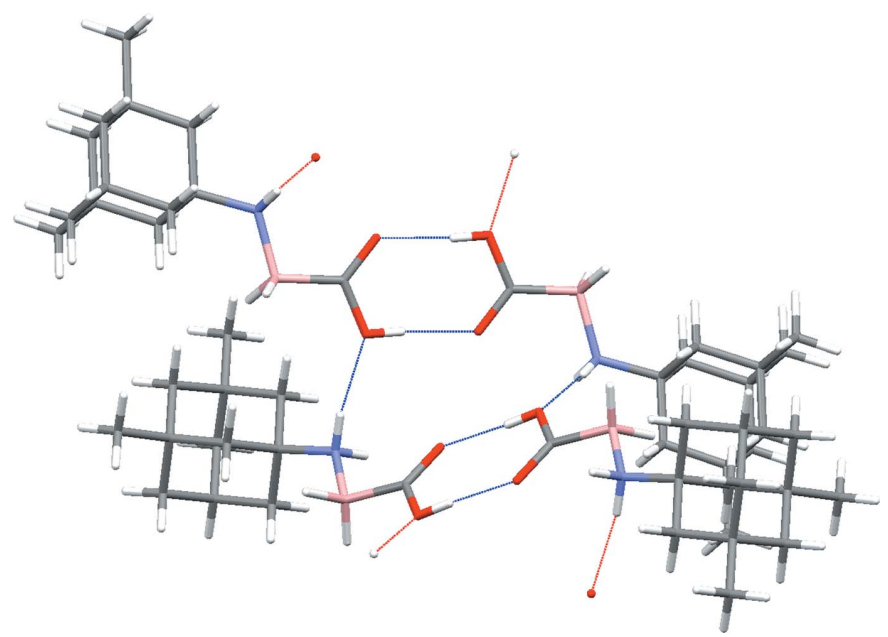

Figure 2

Detail of the hydrogen bonds in (I) showing the carboxylic acid inversion dimers and $\mathrm{N}-\mathrm{H} \cdots \mathrm{O}$ links between dimers. 
(a)

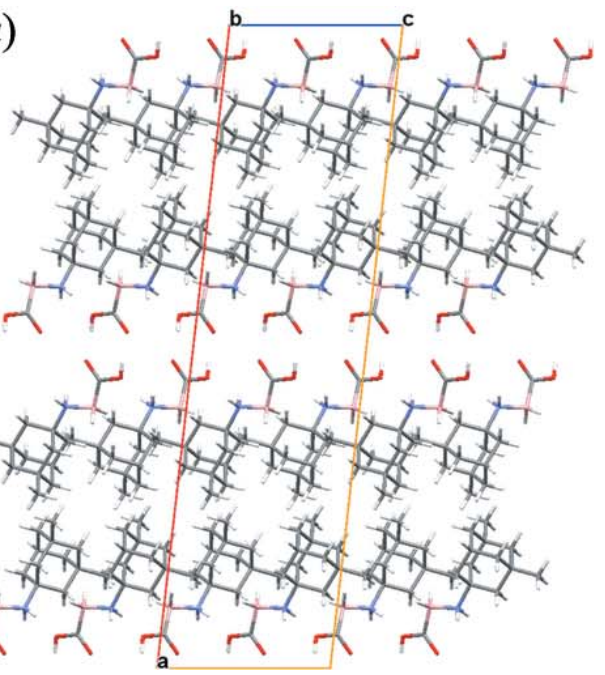

(b)

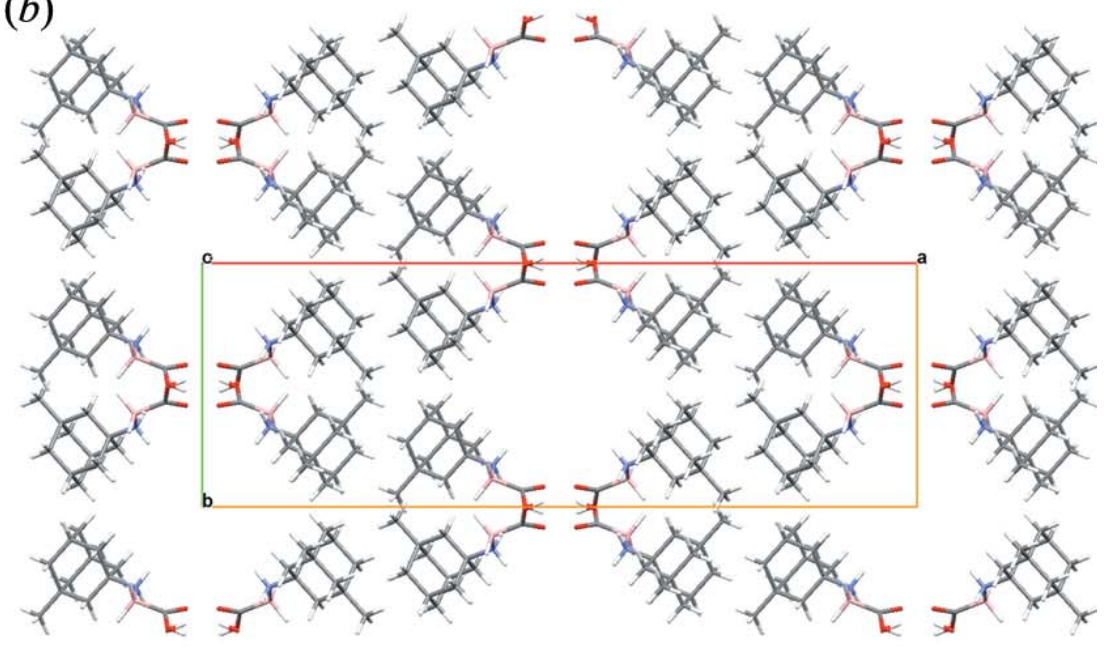

Figure 3

Packing diagrams of (I): (a) A view from the $b$ axis to show aligned hydrogen-bonding dimers. ( $b$ ) A view down the $c$ axis to show the twisted planes.

However, numerous crystal structures of the adamantane cage and its derivatives in various forms have been reported over many years (Nordman \& Schmitkons, 1965; Chacko \& Zand, 1973; SiMa, 2009; Glaser et al., 2011).

\section{Synthesis and crystallization}

Memantine, a derivative of adamantine, was first synthesized by Eli Lilly and Company. In an attempt to modify memantine into memantine-carboxyborane, a reaction scheme as shown in Fig. 4 was carried out. Addition of the carboxyborane moiety to memantine was done in a one-step reaction using an amine-exchange process as previously described (Spielvogel et al., 1980). Trimethylamine carboxyborane (117 mg, $1.0 \mathrm{mmol})$ and memantine (780 mg, $4.4 \mathrm{mmol}$ ) were dissolved in tetrahydrofuran $(8.0 \mathrm{ml})$, and maintained at $328 \mathrm{~K}$ for $24 \mathrm{~h}$ under a nitrogen atmosphere. The solution was concentrated by vacuum distillation and the resulting solid was dissolved in dichloromethane. The product was precipitated from the solvent by using $15 \mathrm{ml}$ of hexane and the white solid crude product $(208 \mathrm{mg}$ ) was filtered. This residue was purified by multiple recrystallization in dichloromethane/hexane to yield a white solid (15 mg, 6.3\%). Crystals suitable for X-ray analysis were prepared by dissolving in toluene and slow cooling of the solution.

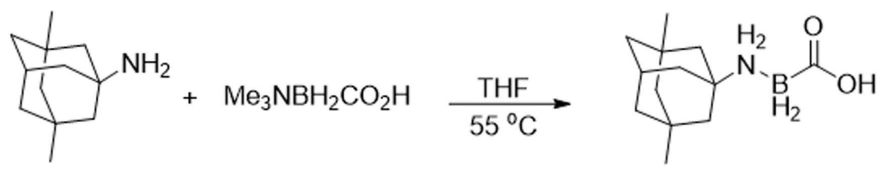

Figure 4

Reaction scheme for the synthesis of (I) through an amine-exchange process.

\section{Refinement}

Crystal data collection and structure refinement details are summarized in Table 2. $\mathrm{H}$ atoms were placed in calculated positions $(\mathrm{O}-\mathrm{H}=0.84, \mathrm{~N}-\mathrm{H}=0.91$ and $\mathrm{C}-\mathrm{H}=0.98-0.99 \AA)$ and refined as riding with $U_{\text {iso }}(\mathrm{eq})=1.5 U_{\text {eq }}(\mathrm{C}$-methyl, $\mathrm{O})$ and $1.2 U_{\text {eq }}(\mathrm{C}, \mathrm{N})$ for all others. The idealized methyl groups at $\mathrm{C} 12$

Table 2

Experimental details.

Crystal data

Chemical formula

$M_{\mathrm{r}}$

Crystal system, space group

Temperature (K)

$a, b, c(\AA)$

$\beta\left({ }^{\circ}\right)$

$V\left(\AA^{3}\right)$

$Z$

Radiation type

$\mu\left(\mathrm{mm}^{-1}\right)$

Crystal size (mm)

Data collection

Diffractometer

Absorption correction

$T_{\min }, T_{\max }$

No. of measured, independent and observed $[I>2 \sigma(I)]$ reflections $(\sin \theta / \lambda)_{\max }\left(\AA^{-1}\right)$

Refinement

$R\left[F^{2}>2 \sigma\left(F^{2}\right)\right], w R\left(F^{2}\right), S$

No. of reflections

No. of parameters

$\mathrm{H}$-atom treatment

$\Delta \rho_{\max }, \Delta \rho_{\min }\left(\mathrm{e} \AA^{-3}\right)$

Computer programs: APEX3 and SAINT (Bruker, 2017), SHELXT (Sheldrick, 2015a), SHELXL2014 (Sheldrick, 2015b) and OLEX2 (Dolomanov et al., 2009).

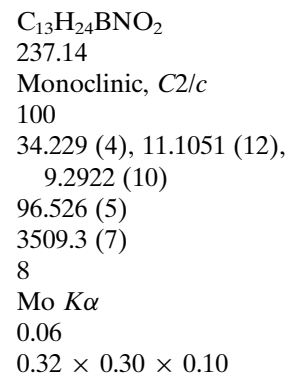

Bruker APEXII Ultra Multi-scan (TWINABS; Bruker, 2012)

$0.300,0.333$

10740, 10740, 8531

0.611
$0.062,0.154,1.04$
10740
166
$\mathrm{H}$ atoms treated by a mixture of independent and constrained refinement
refinement


and $\mathrm{C} 13$ and the idealized tetrahedral $\mathrm{OH}$ group at $\mathrm{O} 1$ were refined as rotating groups. The disordered solvent molecules were treated with the SQUEEZE routine in PLATON (Spek, 2015). The crystal studied was refined as a two-component twin.

\section{Acknowledgements}

Funding for this research was provided by an Institutional Development Award (IDeA) from the National Institute of General Medical Sciences of the National Institutes of Health under grant No. P20GM103395. The content is solely the responsibility of the authors and does not necessarily reflect the official views of the NIH.

\section{Funding information}

Funding for this research was provided by: National Institutes of Health, National Institute of General Medical Sciences (grant No. P20GM103395).

\section{References}

Ayudhya, T. I., Pellechia, P. J. \& Dingra, N. N. (2018). Dalton Trans. 47, 538-543.

Ayudhya, T. I., Raymond, C. C. \& Dingra, N. N. (2017). Dalton Trans. 46, 882-889.

Bruker (2012). TWINABS. Bruker AXS Inc., Madison, Wisconsin, USA.

Bruker (2017). APEX3 and SAINT. Bruker AXS Inc., Madison, Wisconsin, USA.
Bullock, R. (2006). Alzheimer Dis. Assoc. Disord. 20, 23-29.

Chacko, K. K. \& Zand, R. (1973). Acta Cryst. B29, 2681-2686.

Dolomanov, O. V., Bourhis, L. J., Gildea, R. J., Howard, J. A. K. \& Puschmann, H. (2009). J. Appl. Cryst. 42, 339-341.

Donohue, J. \& Goodman, S. H. (1967). Acta Cryst. 22, 352-354.

Gavezzotti, A. (2008). Acta Cryst. B64, 401-403.

Glaser, R., Steinberg, A., Sekutor, M., Rominger, F., Trapp, O. \& Mlinarić-Majerski, K. (2011). Eur. J. Org. Chem. pp. 3500-3506.

Groom, C. R., Bruno, I. J., Lightfoot, M. P. \& Ward, S. C. (2016). Acta Cryst. B72, 171-179.

Lipton, S. A. (2005). Curr. Alzheimer Res. 2, 155-165.

Lou, W.-J., Hu, X.-R. \& Gu, J.-M. (2009). Acta Cryst. E65, o2191.

McInnes, F. J., Anthony, N. G., Kennedy, A. R. \& Wheate, N. J. (2010). Org. Biomol. Chem. 8, 765-773.

Nordman, C. E. \& Schmitkons, D. L. (1965). Acta Cryst. 18, 764-767.

Olivares, D., Deshpande, V. K., Shi, Y., Lahiri, D. K., Greig, N. H., Rogers, J. T. \& Huang, X. (2012). Curr. Alzheimer Res. 9, 746-758.

Parsons, C. G., Stöffler, A. \& Danysz, W. (2007). Neuropharmacology, 53, 699-723.

Plosker, G. L. (2015). Drugs, 75, 887-897.

Rana, G., Vyakaranam, K., Zheng, C., Li, S., Spielvogel, B. F. \& Hosmane, N. S. (2002). Main Group Met. Chem. 25, 107-108.

Sheldrick, G. M. (2015a). Acta Cryst. A71, 3-8.

Sheldrick, G. M. (2015b). Acta Cryst. C71, 3-8.

SiMa, W. (2009). Acta Cryst. E65, o2492.

Spek, A. L. (2015). Acta Cryst. C71, 9-18.

Spielvogel, B. F., Das, M. K., McPhail, A. T., Onan, K. D. \& Hall, I. H. (1980). J. Am. Chem. Soc. 102, 6343-6344.

Spielvogel, B. F., Wojnowich, L., Das, M. K., McPhail, A. T. \& Hargrave, K. D. (1976). J. Am. Chem. Soc. 98, 5702-5703.

Vyakaranam, K., Rana, G., Chong, G., Zheng, S. L., Spielvogel, B. F. \& Hosmane, N. S. (2002). Main Group Met. Chem. 25, 181-182. 


\section{supporting information}

Acta Cryst. (2019). E75, 543-546 [https://doi.org/10.1107/S2056989019004092]

\section{Crystal structure of memantine-carboxyborane}

\section{Theppawut I. Ayudhya, Arnold L. Rheingold and Nin N. Dingra}

Computing details

Data collection: APEX3 (Bruker, 2017); cell refinement: SAINT (Bruker, 2017); data reduction: SAINT (Bruker, 2017); program(s) used to solve structure: SHELXT (Sheldrick, 2015a); program(s) used to refine structure: SHELXL2014 (Sheldrick, 2015b); molecular graphics: OLEX2 (Dolomanov et al., 2009); software used to prepare material for publication: OLEX2 (Dolomanov et al., 2009).

3,5-Dimethyladamantanylamine-boranecarboxylic acid

Crystal data

$\mathrm{C}_{13} \mathrm{H}_{24} \mathrm{BNO}_{2}$

$M_{r}=237.14$

Monoclinic, $C 2 / c$

$a=34.229(4) \AA$

$b=11.1051(12) \AA$

$c=9.2922(10) \AA$

$\beta=96.526(5)^{\circ}$

$V=3509.3(7) \AA^{3}$

$Z=8$

Data collection

Bruker APEXII Ultra diffractometer

Radiation source: Micro Focus Rotating Anode, Bruker TXS

Double Bounce Multilayer Mirrors monochromator

Detector resolution: 8.258 pixels $\mathrm{mm}^{-1}$ $\varphi$ and $\omega$ scans
$F(000)=1040$

$D_{\mathrm{x}}=0.898 \mathrm{Mg} \mathrm{m}^{-3}$

Mo $K \alpha$ radiation, $\lambda=0.71073 \AA$

Cell parameters from 1799 reflections

$\theta=2.9-25.6^{\circ}$

$\mu=0.06 \mathrm{~mm}^{-1}$

$T=100 \mathrm{~K}$

Plate, colourless

$0.32 \times 0.30 \times 0.10 \mathrm{~mm}$

\section{Refinement}

Refinement on $F^{2}$

Least-squares matrix: full

$R\left[F^{2}>2 \sigma\left(F^{2}\right)\right]=0.062$

$w R\left(F^{2}\right)=0.154$

$S=1.04$

10740 reflections

166 parameters

0 restraints

Primary atom site location: dual

\footnotetext{
Absorption correction: multi-scan (TWINABS; Bruker, 2012)

$T_{\min }=0.300, T_{\max }=0.333$

10740 measured reflections

10740 independent reflections

8531 reflections with $I>2 \sigma(I)$

$\theta_{\text {max }}=25.7^{\circ}, \theta_{\text {min }}=1.2^{\circ}$

$h=-41 \rightarrow 41$

$k=-13 \rightarrow 13$

$l=-11 \rightarrow 11$
}

Hydrogen site location: mixed

$\mathrm{H}$ atoms treated by a mixture of independent and constrained refinement

$w=1 /\left[\sigma^{2}\left(F_{\mathrm{o}}^{2}\right)+(0.0598 P)^{2}+6.9836 P\right]$ where $P=\left(F_{\mathrm{o}}^{2}+2 F_{\mathrm{c}}{ }^{2}\right) / 3$

$(\Delta / \sigma)_{\max }<0.001$

$\Delta \rho_{\max }=0.94 \mathrm{e} \AA^{-3}$

$\Delta \rho_{\min }=-0.24$ e $\AA^{-3}$ 


\section{Special details}

Geometry. All esds (except the esd in the dihedral angle between two 1.s. planes) are estimated using the full covariance matrix. The cell esds are taken into account individually in the estimation of esds in distances, angles and torsion angles; correlations between esds in cell parameters are only used when they are defined by crystal symmetry. An approximate (isotropic) treatment of cell esds is used for estimating esds involving l.s. planes.

Refinement. Refined as a two-component twin.

Fractional atomic coordinates and isotropic or equivalent isotropic displacement parameters $\left(\AA^{2}\right)$

\begin{tabular}{|c|c|c|c|c|}
\hline & $x$ & $y$ & $z$ & $U_{\text {iso }} * / U_{\text {eq }}$ \\
\hline O1 & $0.54884(6)$ & $0.9933(2)$ & $1.1021(3)$ & $0.0296(6)$ \\
\hline H1 & 0.5259 & 1.0205 & 1.0994 & $0.044 *$ \\
\hline $\mathrm{O} 2$ & $0.52308(7)$ & $0.9168(2)$ & $0.8929(2)$ & $0.0373(7)$ \\
\hline N1 & $0.59334(7)$ & $0.8289(2)$ & 0.8033 & $0.0214(6)$ \\
\hline $\mathrm{H} 1 \mathrm{~A}$ & 0.5763 & 0.7656 & 0.7974 & $0.026^{*}$ \\
\hline H1B & 0.5825 & 0.8865 & 0.7416 & $0.026^{*}$ \\
\hline C9 & $0.61930(9)$ & 0.7507 & $0.5864(3)$ & $0.0203(7)$ \\
\hline H9A & 0.5993 & 0.6859 & 0.5806 & $0.024 *$ \\
\hline H9B & 0.6077 & 0.8209 & 0.5313 & $0.024^{*}$ \\
\hline $\mathrm{C} 2$ & $0.63037(8)$ & $0.7867(3)$ & 0.7461 & $0.0172(7)$ \\
\hline $\mathrm{C} 10$ & $0.64666(9)$ & $0.6768(3)$ & $0.8315(4)$ & $0.0224(7)$ \\
\hline $\mathrm{H} 10 \mathrm{~A}$ & 0.6267 & 0.6120 & 0.8257 & $0.027 *$ \\
\hline $\mathrm{H} 10 \mathrm{~B}$ & 0.6536 & 0.6983 & 0.9346 & $0.027^{*}$ \\
\hline $\mathrm{C} 8$ & $0.65553(9)$ & $0.7071(3)$ & $0.5192(3)$ & $0.0213(7)$ \\
\hline C11 & $0.68651(9)$ & $0.8083(3)$ & $0.5324(4)$ & $0.0241(8)$ \\
\hline H11A & 0.7103 & 0.7806 & 0.4906 & $0.029 *$ \\
\hline H11B & 0.6759 & 0.8793 & 0.4764 & $0.029 *$ \\
\hline C6 & $0.68384(9)$ & 0.6335 & $0.7642(4)$ & $0.0229(8)$ \\
\hline H6 & 0.6951 & 0.5618 & 0.8193 & $0.027^{*}$ \\
\hline $\mathrm{C} 1$ & $0.55232(10)$ & $0.9311(3)$ & $0.9806(4)$ & $0.0219(8)$ \\
\hline $\mathrm{C} 4$ & $0.69762(9)$ & $0.8445(3)$ & 0.6898 & $0.0243(8)$ \\
\hline $\mathrm{C} 3$ & $0.66090(9)$ & $0.8865(3)$ & $0.7553(4)$ & $0.0222(7)$ \\
\hline $\mathrm{H} 3 \mathrm{~A}$ & 0.6681 & 0.9095 & 0.8578 & $0.027^{*}$ \\
\hline H3B & 0.6498 & 0.9583 & 0.7023 & $0.027^{*}$ \\
\hline $\mathrm{C} 7$ & $0.67253(9)$ & $0.5977(3)$ & $0.6057(4)$ & $0.0225(7)$ \\
\hline H7A & 0.6528 & 0.5323 & 0.6002 & $0.027^{*}$ \\
\hline H7B & 0.6960 & 0.5676 & 0.5639 & $0.027 *$ \\
\hline $\mathrm{C} 5$ & $0.71424(9)$ & $0.7332(3)$ & $0.7751(4)$ & $0.0277(8)$ \\
\hline $\mathrm{H} 5 \mathrm{~A}$ & 0.7383 & 0.7050 & 0.7354 & $0.033^{*}$ \\
\hline H5B & 0.7214 & 0.7551 & 0.8780 & $0.033 *$ \\
\hline $\mathrm{C} 13$ & $0.64387(10)$ & $0.6749(3)$ & $0.3590(4)$ & $0.0298(9)$ \\
\hline $\mathrm{H} 13 \mathrm{~A}$ & 0.6244 & 0.6099 & 0.3522 & $0.045^{*}$ \\
\hline H13B & 0.6672 & 0.6484 & 0.3157 & $0.045 *$ \\
\hline $\mathrm{H} 13 \mathrm{C}$ & 0.6326 & 0.7458 & 0.3071 & $0.045^{*}$ \\
\hline C12 & $0.72819(10)$ & 0.9460 & $0.6973(5)$ & $0.0397(10)$ \\
\hline $\mathrm{H} 12 \mathrm{~A}$ & 0.7515 & 0.9185 & 0.6543 & $0.060^{*}$ \\
\hline H12B & 0.7358 & 0.9683 & 0.7988 & $0.060 *$ \\
\hline $\mathrm{H} 12 \mathrm{C}$ & 0.7169 & 1.0162 & 0.6438 & $0.060^{*}$ \\
\hline
\end{tabular}




$\begin{array}{lllll}\text { B1 } & 0.59514(12) & 0.8823(4) & 0.9644(4) & 0.0295(10) \\ \text { H1C } & 0.6174(10) & 0.964(3) & 0.966(4) & 0.042(10)^{*} \\ \text { H1D } & 0.6026(11) & 0.810(3) & 1.038(4) & 0.050(11)^{*}\end{array}$

Atomic displacement parameters $\left(\AA^{2}\right)$

\begin{tabular}{lllllll}
\hline & $U^{11}$ & $U^{22}$ & $U^{33}$ & $U^{12}$ & $U^{13}$ & $U^{23}$ \\
\hline O1 & $0.0235(12)$ & $0.0335(13)$ & $0.0342(14)$ & $-0.0008(11)$ & $0.0140(12)$ & $-0.0136(11)$ \\
O2 & $0.0334(14)$ & $0.0620(17)$ & $0.0167(12)$ & $0.0240(13)$ & $0.0038(12)$ & $-0.0048(13)$ \\
N1 & $0.0202(14)$ & $0.0169(13)$ & $0.0280(15)$ & $0.0034(11)$ & $0.0062(12)$ & $0.0043(12)$ \\
C9 & $0.0237(18)$ & $0.0150(16)$ & $0.0222(17)$ & $0.0018(13)$ & $0.0026(15)$ & $0.0029(15)$ \\
C2 & $0.0149(16)$ & $0.0182(16)$ & $0.0190(16)$ & $0.0039(13)$ & $0.0043(14)$ & $-0.0018(14)$ \\
C10 & $0.0236(18)$ & $0.0213(17)$ & $0.0230(17)$ & $0.0000(14)$ & $0.0062(15)$ & $0.0018(15)$ \\
C8 & $0.0238(18)$ & $0.0177(16)$ & $0.0227(18)$ & $0.0022(14)$ & $0.0046(15)$ & $0.0007(15)$ \\
C11 & $0.0225(18)$ & $0.0206(17)$ & $0.032(2)$ & $0.0023(14)$ & $0.0134(16)$ & $0.0027(16)$ \\
C6 & $0.0221(17)$ & $0.0197(16)$ & $0.0268(18)$ & $0.0032(14)$ & $0.0027(15)$ & $0.0045(15)$ \\
C1 & $0.030(2)$ & $0.0181(17)$ & $0.0183(17)$ & $0.0013(14)$ & $0.0046(16)$ & $0.0034(15)$ \\
C4 & $0.0172(17)$ & $0.0215(17)$ & $0.035(2)$ & $-0.0027(14)$ & $0.0082(16)$ & $-0.0072(16)$ \\
C3 & $0.0238(18)$ & $0.0193(17)$ & $0.0235(17)$ & $-0.0012(14)$ & $0.0024(15)$ & $-0.0002(15)$ \\
C7 & $0.0214(18)$ & $0.0187(16)$ & $0.0287(17)$ & $0.0021(14)$ & $0.0079(16)$ & $-0.0043(16)$ \\
C5 & $0.0170(17)$ & $0.037(2)$ & $0.0290(19)$ & $0.0053(15)$ & $0.0007(16)$ & $-0.0096(18)$ \\
C13 & $0.033(2)$ & $0.033(2)$ & $0.023(2)$ & $0.0073(17)$ & $0.0038(16)$ & $0.0011(17)$ \\
C12 & $0.027(2)$ & $0.036(2)$ & $0.058(3)$ & $-0.0085(17)$ & $0.012(2)$ & $-0.012(2)$ \\
B1 & $0.025(2)$ & $0.037(3)$ & $0.028(2)$ & $0.0001(19)$ & $0.0062(18)$ & $-0.011(2)$ \\
& & & & & \\
\hline
\end{tabular}

Geometric parameters $\left(\AA,{ }^{\circ}\right)$

\begin{tabular}{llll}
\hline $\mathrm{O} 1-\mathrm{H} 1$ & 0.8400 & $\mathrm{C} 6-\mathrm{H} 6$ & 1.0000 \\
$\mathrm{O} 1-\mathrm{C} 1$ & $1.340(4)$ & $\mathrm{C} 6-\mathrm{C} 7$ & $1.532(5)$ \\
$\mathrm{O} 2-\mathrm{C} 1$ & $1.227(4)$ & $\mathrm{C} 6-\mathrm{C} 5$ & $1.515(4)$ \\
$\mathrm{N} 1-\mathrm{H} 1 \mathrm{~A}$ & 0.9100 & $\mathrm{C} 1-\mathrm{B} 1$ & $1.586(5)$ \\
$\mathrm{N} 1-\mathrm{H} 1 \mathrm{~B}$ & 0.9100 & $\mathrm{C} 4-\mathrm{C} 3$ & $1.530(4)$ \\
$\mathrm{N} 1-\mathrm{C} 2$ & $1.504(4)$ & $\mathrm{C} 4-\mathrm{C} 5$ & $1.541(4)$ \\
$\mathrm{N} 1-\mathrm{B} 1$ & $1.605(5)$ & $\mathrm{C} 4-\mathrm{C} 12$ & $1.535(4)$ \\
$\mathrm{C} 9-\mathrm{H} 9 \mathrm{~A}$ & 0.9900 & $\mathrm{C} 3-\mathrm{H} 3 \mathrm{~A}$ & 0.9900 \\
$\mathrm{C} 9-\mathrm{H} 9 \mathrm{~B}$ & 0.9900 & $\mathrm{C} 3-\mathrm{H} 3 \mathrm{~B}$ & 0.9900 \\
$\mathrm{C} 9-\mathrm{C} 2$ & $1.542(4)$ & $\mathrm{C} 7-\mathrm{H} 7 \mathrm{~A}$ & 0.9900 \\
$\mathrm{C} 9-\mathrm{C} 8$ & $1.530(4)$ & $\mathrm{C} 7-\mathrm{H} 7 \mathrm{~B}$ & 0.9900 \\
$\mathrm{C} 2-\mathrm{C} 10$ & $1.525(4)$ & $\mathrm{C} 5-\mathrm{H} 5 \mathrm{~A}$ & 0.9900 \\
$\mathrm{C} 2-\mathrm{C} 3$ & $1.520(4)$ & $\mathrm{C} 5-\mathrm{H} 5 \mathrm{~B}$ & 0.9900 \\
$\mathrm{C} 10-\mathrm{H} 10 \mathrm{~A}$ & 0.9900 & $\mathrm{C} 13-\mathrm{H} 13 \mathrm{~A}$ & 0.9800 \\
$\mathrm{C} 10-\mathrm{H} 10 \mathrm{~B}$ & 0.9900 & $\mathrm{C} 13-\mathrm{H} 13 \mathrm{~B}$ & 0.9800 \\
$\mathrm{C} 10-\mathrm{C} 6$ & $1.557(4)$ & $\mathrm{C} 13-\mathrm{H} 13 \mathrm{C}$ & 0.9800 \\
$\mathrm{C} 8-\mathrm{C} 11$ & $1.541(4)$ & $\mathrm{C} 12-\mathrm{H} 12 \mathrm{~A}$ & 0.9800 \\
$\mathrm{C} 8-\mathrm{C} 7$ & $1.535(4)$ & $\mathrm{C} 12-\mathrm{H} 12 \mathrm{~B}$ & 0.9800 \\
$\mathrm{C} 8-\mathrm{C} 13$ & $1.539(5)$ & $\mathrm{C} 12-\mathrm{H} 12 \mathrm{C}$ & 0.9800 \\
$\mathrm{C} 11-\mathrm{H} 11 \mathrm{~A}$ & 0.9900 & $\mathrm{~B} 1-\mathrm{H} 1 \mathrm{C}$ & $1.19(3)$ \\
$\mathrm{C} 11-\mathrm{H} 11 \mathrm{~B}$ & 0.9900 & $\mathrm{~B} 1-\mathrm{H} 1 \mathrm{D}$ & $1.07(4)$
\end{tabular}




$$
\mathrm{C} 11-\mathrm{C} 4
$$

$\mathrm{C} 1-\mathrm{O} 1-\mathrm{H} 1$

H1A-N1-H1B

$\mathrm{C} 2-\mathrm{N} 1-\mathrm{H} 1 \mathrm{~A}$

$\mathrm{C} 2-\mathrm{N} 1-\mathrm{H} 1 \mathrm{~B}$

$\mathrm{C} 2-\mathrm{N} 1-\mathrm{B} 1$

$\mathrm{B} 1-\mathrm{N} 1-\mathrm{H} 1 \mathrm{~A}$

$\mathrm{B} 1-\mathrm{N} 1-\mathrm{H} 1 \mathrm{~B}$

$\mathrm{H} 9 \mathrm{~A}-\mathrm{C} 9-\mathrm{H} 9 \mathrm{~B}$

$\mathrm{C} 2-\mathrm{C} 9-\mathrm{H} 9 \mathrm{~A}$

$\mathrm{C} 2-\mathrm{C} 9-\mathrm{H} 9 \mathrm{~B}$

$\mathrm{C} 8-\mathrm{C} 9-\mathrm{H} 9 \mathrm{~A}$

C $8-\mathrm{C} 9-\mathrm{H} 9 \mathrm{~B}$

$\mathrm{C} 8-\mathrm{C} 9-\mathrm{C} 2$

$\mathrm{N} 1-\mathrm{C} 2-\mathrm{C} 9$

$\mathrm{N} 1-\mathrm{C} 2-\mathrm{C} 10$

$\mathrm{N} 1-\mathrm{C} 2-\mathrm{C} 3$

$\mathrm{C} 10-\mathrm{C} 2-\mathrm{C} 9$

$\mathrm{C} 3-\mathrm{C} 2-\mathrm{C} 9$

$\mathrm{C} 3-\mathrm{C} 2-\mathrm{C} 10$

$\mathrm{C} 2-\mathrm{C} 10-\mathrm{H} 10 \mathrm{~A}$

$\mathrm{C} 2-\mathrm{C} 10-\mathrm{H} 10 \mathrm{~B}$

$\mathrm{C} 2-\mathrm{C} 10-\mathrm{C} 6$

$\mathrm{H} 10 \mathrm{~A}-\mathrm{C} 10-\mathrm{H} 10 \mathrm{~B}$

C6- C10-H10A

C6- C10-H10B

$\mathrm{C} 9-\mathrm{C} 8-\mathrm{C} 11$

$\mathrm{C} 9-\mathrm{C} 8-\mathrm{C} 7$

$\mathrm{C} 9-\mathrm{C} 8-\mathrm{C} 13$

$\mathrm{C} 7-\mathrm{C} 8-\mathrm{C} 11$

$\mathrm{C} 7-\mathrm{C} 8-\mathrm{C} 13$

$\mathrm{C} 13-\mathrm{C} 8-\mathrm{C} 11$

C8-C11-H11A

C8-C11-H11B

H11A-C11-H11B

C4- $\mathrm{C} 11-\mathrm{C} 8$

C4-C11-H11A

C4-C11-H11B

C10- 6 6- 6

$\mathrm{C} 7-\mathrm{C} 6-\mathrm{C} 10$

$\mathrm{C} 7-\mathrm{C} 6-\mathrm{H} 6$

$\mathrm{C} 5-\mathrm{C} 6-\mathrm{C} 10$

$\mathrm{C} 5-\mathrm{C} 6-\mathrm{H} 6$

C5-C6-C7

$\mathrm{O} 1-\mathrm{C} 1-\mathrm{B} 1$
$1.523(5)$

109.5

106.9

107.3

107.3

120.0 (3)

107.3

107.3

108.1

109.5

109.5

109.5

109.5

110.7 (2)

$107.2(2)$

$109.8(2)$

$110.8(2)$

$109.2(2)$

$109.6(3)$

$110.2(2)$

110.1

110.1

$107.8(3)$

108.5

110.1

110.1

$108.6(3)$

$108.3(3)$

$109.6(3)$

$108.6(3)$

$111.4(3)$

$110.3(3)$

109.4

109.4

108.0

$111.3(3)$

109.4

109.4

109.0

$109.7(3)$

109.0

$109.5(3)$

109.0

$110.5(3)$

$115.4(3)$

$\begin{array}{ll}\mathrm{O} 2-\mathrm{C} 1-\mathrm{O} 1 & 118.8(3) \\ \mathrm{O} 2-\mathrm{C} 1-\mathrm{B} 1 & 125.8(3) \\ \mathrm{C} 11-\mathrm{C} 4-\mathrm{C} 3 & 109.6(3) \\ \mathrm{C} 11-\mathrm{C} 4-\mathrm{C} 5 & 108.6(3) \\ \mathrm{C} 11-\mathrm{C} 4-\mathrm{C} 12 & 109.4(3) \\ \mathrm{C} 3-\mathrm{C} 4-\mathrm{C} 5 & 108.2(3) \\ \mathrm{C} 3-\mathrm{C} 4-\mathrm{C} 12 & 110.1(3) \\ \mathrm{C} 12-\mathrm{C} 4-\mathrm{C} 5 & 110.8(3) \\ \mathrm{C} 2-\mathrm{C} 3-\mathrm{C} 4 & 110.2(2) \\ \mathrm{C} 2-\mathrm{C} 3-\mathrm{H} 3 \mathrm{~A} & 109.6 \\ \mathrm{C} 2-\mathrm{C} 3-\mathrm{H} 3 \mathrm{~B} & 109.6 \\ \mathrm{C} 4-\mathrm{C} 3-\mathrm{H} 3 \mathrm{~A} & 109.6 \\ \mathrm{C} 4-\mathrm{C} 3-\mathrm{H} 3 \mathrm{~B} & 109.6 \\ \mathrm{H} 3 \mathrm{~A}-\mathrm{C} 3-\mathrm{H} 3 \mathrm{~B} & 108.1 \\ \mathrm{C} 8-\mathrm{C} 7-\mathrm{H} 7 \mathrm{~A} & 109.7 \\ \mathrm{C} 8-\mathrm{C} 7-\mathrm{H} 7 \mathrm{~B} & 109.7 \\ \mathrm{C} 6-\mathrm{C} 7-\mathrm{C} 8 & 109.7(3) \\ \mathrm{C} 6-\mathrm{C} 7-\mathrm{H} 7 \mathrm{~A} & 109.7 \\ \mathrm{C} 6-\mathrm{C} 7-\mathrm{H} 7 \mathrm{~B} & 109.7 \\ \mathrm{H} 7 \mathrm{~A}-\mathrm{C} 7-\mathrm{H} 7 \mathrm{~B} & 108.2 \\ \mathrm{C} 6-\mathrm{C} 5-\mathrm{C} 4 & 109.9(3) \\ \mathrm{C} 6-\mathrm{C} 5-\mathrm{H} 5 \mathrm{~A} & 109.7\end{array}$

$\mathrm{C} 6-\mathrm{C} 5-\mathrm{H} 5 \mathrm{~A} \quad 109.7$

C6- C5-H5B 109.7

$\mathrm{C} 4-\mathrm{C} 5-\mathrm{H} 5 \mathrm{~A} \quad 109.7$

$\mathrm{C} 4-\mathrm{C} 5-\mathrm{H} 5 \mathrm{~B} \quad 109.7$

$\mathrm{H} 5 \mathrm{~A}-\mathrm{C} 5-\mathrm{H} 5 \mathrm{~B} \quad 108.2$

$\mathrm{C} 8-\mathrm{C} 13-\mathrm{H} 13 \mathrm{~A} \quad 109.5$

$\mathrm{C} 8-\mathrm{C} 13-\mathrm{H} 13 \mathrm{~B} \quad 109.5$

$\mathrm{C} 8-\mathrm{C} 13-\mathrm{H} 13 \mathrm{C} \quad 109.5$

$\mathrm{H} 13 \mathrm{~A}-\mathrm{C} 13-\mathrm{H} 13 \mathrm{~B} \quad 109.5$

$\mathrm{H} 13 \mathrm{~A}-\mathrm{C} 13-\mathrm{H} 13 \mathrm{C} \quad 109.5$

$\mathrm{H} 13 \mathrm{~B}-\mathrm{C} 13-\mathrm{H} 13 \mathrm{C} \quad 109.5$

$\mathrm{C} 4-\mathrm{C} 12-\mathrm{H} 12 \mathrm{~A} \quad 109.5$

$\mathrm{C} 4-\mathrm{C} 12-\mathrm{H} 12 \mathrm{~B} \quad 109.5$

$\mathrm{C} 4-\mathrm{C} 12-\mathrm{H} 12 \mathrm{C} \quad 109.5$

$\mathrm{H} 12 \mathrm{~A}-\mathrm{C} 12-\mathrm{H} 12 \mathrm{~B} \quad 109.5$

$\mathrm{H} 12 \mathrm{~A}-\mathrm{C} 12-\mathrm{H} 12 \mathrm{C} \quad 109.5$

$\mathrm{H} 12 \mathrm{~B}-\mathrm{C} 12-\mathrm{H} 12 \mathrm{C} \quad 109.5$

$\mathrm{N} 1-\mathrm{B} 1-\mathrm{H} 1 \mathrm{C} \quad 104.6(18)$

$\mathrm{N} 1-\mathrm{B} 1-\mathrm{H} 1 \mathrm{D} \quad 107(2)$

$\mathrm{C} 1-\mathrm{B} 1-\mathrm{N} 1$

$\mathrm{C} 1-\mathrm{B} 1-\mathrm{H} 1 \mathrm{C} \quad 109.7(16)$

$\mathrm{C} 1-\mathrm{B} 1-\mathrm{H} 1 \mathrm{D}$

H1C-B1-H1D 118 (3) 
Hydrogen-bond geometry $\left(A,{ }^{\circ}\right)$

\begin{tabular}{lllll}
\hline$D-\mathrm{H} \cdots A$ & $D-\mathrm{H}$ & $\mathrm{H} \cdots A$ & $D \cdots A$ & $D-\mathrm{H} \cdots A$ \\
\hline $\mathrm{O} 1-\mathrm{H} 1 \cdots \mathrm{O} 2^{\mathrm{i}}$ & 0.84 & 1.82 & $2.662(3)$ & 176 \\
$\mathrm{~N} 1-\mathrm{H} 1 B^{\cdots} \cdots{ }^{\mathrm{ii}}$ & 0.91 & 2.11 & $3.011(3)$ & 171 \\
\hline
\end{tabular}

Symmetry codes: (i) $-x+1,-y+2,-z+2$; (ii) $x,-y+2, z-1 / 2$. 